\title{
Object Detection and Sorting using IoT
}

\author{
Mr. Dharmesh Dhabliya, Ms. Ritika Dhabalia \\ $R \& D$ Department, Yashika Publications Maharashtra India \\ dharmeshdhabliya@gmail.com
}

\begin{tabular}{|c|c|}
\hline Article History & Abstract \\
\hline $\begin{array}{l}\text { Article Submission } \\
22 \text { June } 2014 \\
\text { Revised Submission } \\
7 \text { September } 2014 \\
\text { Article Accepted } \\
28 \text { November } 2014 \\
\text { Article Published } \\
31^{\text {st }} \text { December } 2014\end{array}$ & $\begin{array}{l}\text { Color based object sorting has a significant impact in food and processing } \\
\text { Industries. Hand picking process in sorting the huge number of objects in industry is } \\
\text { very common and laborious task, and time consuming as well, which needs many } \\
\text { labors and this conventional method is prone to error. The proposed work aims to } \\
\text { replace the hand-picking process by Industrial Internet of Things. The goal of the } \\
\text { technique is to sort and count the objects in to different bins accord to their color. A } \\
\text { Color sensor, TCS } 230 \text { will identify the object and with the help of motors they are } \\
\text { made to drop into different bins. The identification of the object is made with the help } \\
\text { of frequency concept. As it known that different colors have different wave lengths, so } \\
\text { are the different frequencies (f=c/ } \lambda \text { ). For each frequency, the motor rotates to } \\
\text { different angles and thus container is attached to motor is also made to rotate to a } \\
\text { certain angle, and the object is made to drop into the bin by a jerk. This action } \\
\text { details regarding number of objects manufactured are sent to the IoT server, where } \\
\text { the vendor and customer will know the details remotely. This proposed work finds a } \\
\text { wide range of usage in fruit industry (to pick the unripen fruit), in candy industry, in } \\
\text { grain industry (to remove the black stones from the grains), in recycling industry. } \\
\text { Keywords: IoT, TCS230, Thingspeak, Wireless HART }\end{array}$ \\
\hline
\end{tabular}

\section{Introduction}

The automation in industries prove that machines can perform highly repetitive tasks with better efficiency. Certain factors like Work fatigue can reduced performance, and cause challenges in maintaining product quality when humans are involved for long time. Human beings who are performing scrutiny to differentiate objects based on color repeatedly may eventually fail to recognize the color of product leading to error. To improve the efficiency of manufacturing system, automating many of the tasks in the industries is the clear solution. The purpose of an automation model is to fabricate and implement a system which automatically segregate products based on their color. The proposed automation system contains conveyor belt, color sensor, and DC motor. A Microcontroller is used to integrate and control the various activities of the proposed automation system. To reduce human efforts on mechanical working, different types of sorting machines are being developed. The conventional machines are costly to afford, and the fabrication process is complicated. IIoT based automation system in color sensing and object identification industries turn to be a better solution.

\section{Related Work}

To cater fast processing in the current industrial trend and competition, an automation system with reduced error and increased reliability based on classification of products seem to be an ideal solution to speed up the production in any kind of industry and its needs. By developing such sorting systems, the production rate of the manufacturing industry has been increased since the sorting systems replace human. [1]. The author proposed computer vision to detect the fault in final stage of the manufacturing process in ceramic tile attracts more research as it is time consuming. To spot the defects in the textured surface, there arise a standard need for automatic sorting and detection process. This kind of automation will play a major role in testing phase of the 
product and will attract more research. The author delineates the ease to the tile manufacturing industry. As the process involves sorting of faulty tiles [2].

Visual inspection influences the final stage of production ceramic tiles manufacturing process computer vision is used in the problem of automatic inspection of ceramic tiles vision applications attract large research interest in detection of defects in textured surface. It is an important area of automatic industrial inspection that has been largely overlooked by the recent wave of research in machine. The next process involves categorization of typical tile defects [3]. In any manufacturing industry, organizing the final product is a tedious process. The conventional manual sorting cause inconsistency in delivering and packing. The author proposes a working prototype fabricated for automatic delineating of objects based on the color. A well-known TCS230 sensor was employed to ascertain the color of the product. A microcontroller was employed to control the entire sorting and detection process. TCS230 sensor performs frequency analysis of the output for the identification of the color of the product. A pair of conveyor belts used is controlled by individual DC motors. Each pair of conveyor belts perform the following work: positioning the product to be analyzed by the color sensor, moving the container by using separated compartments, in order to segregate the products. The experimental results show that the proposed prototype will cater the needs for enhancing the production in terms of quantity and quality for the development of manufacturing sectors [4].

The authors formulate color sorters that can be used in agricultural machineries that involves sorting of rice, beans and peanuts. The industrial version of the same involve sorting of colors for quartz, sand, fabric granule, colored nuts and bolts that are used in many fabrication industries of agricultural sector. It reduces the human effort, labor and cost. Thus, increasing the speed and efficiency of sorting process to take care of the current and future manufacturing industrial needs. A Robotic arm controlled by microcontroller performs most of the required operation. A light intensity-to-frequency converter operation will differentiate the color and produce an equivalent signal with varying frequency to map the desired object. The fabricated Robotic arm consists of a pair of DC motors [5]. In any manufacturing industry, sorting of products based on variety of parameters attracts more research. To ease the process, color sorting method is proposed by the author. Here the authors propose construction of Object Sorting Robot using image processing. An algorithm to differentiate objects based on wide variety of criteria needed for manufacturing industry has been formulated. This picks and place robot monitor and control the entire process in sequence as per the algorithm. The idea involves capturing the realtime image by using a high-resolution webcam. Then object sorting image processing algorithm is used for identification of color and information out of it. This information is used to pick the desired object based on color and place at position. The proposed work deals with an automated material handling system. [6]

It transfers the different colored objects to different containers for several number of times [7][8]. In mass production industry, there arise a need for a smarter intelligent system to organize the final products. The conventional object sorting system requires more human labor. The author discusses about sorting of the objects based on their contour of the image that defines the shape and color. The entire sequence of steps of results in our desired output of sorting out objects automatically.[9][10].

\section{System Model}

A 5V supply is fed to the Servo motor to initiate the rotation. To sense the color of the object, it holds the ball with the aid of a wooden plate. The Color sensor reflects the light based on frequency. The color sensor TCS230 has 4 color filters for green, red, blue and black (no color), which is opted by programming the microcontroller. The frequency output from color sensor depends on the texture, contour and color of the selected object. Based on the microcontroller output, the data is loaded in thingspeak server and customized for user need. The proposed hardware architecture is shown in figure 1. 


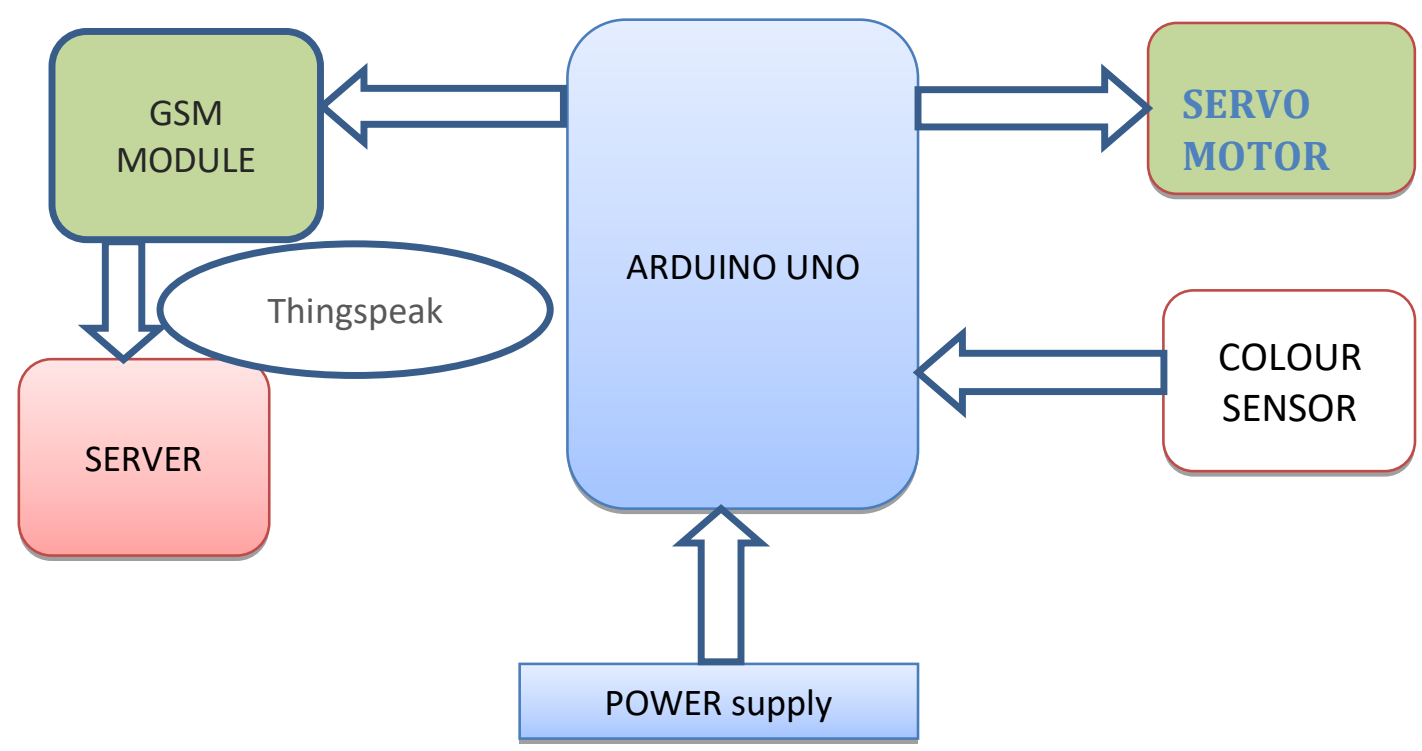

Fig 1: Hardware Architecture of the Proposed Method

A photo diode configuration is set by the frequency of the output sensor that differentiates between the presence and absence of the object. The data output from the thingspeak server purely depends on the 4 filters for different filter configuration. The colour segregation process involves the mode and the dimension of the object under test. Multiple baskets are used in different positions to grab the sensed object. After producing the output, the second servo motor has been offered mobility so that desired items fall into respective basket.

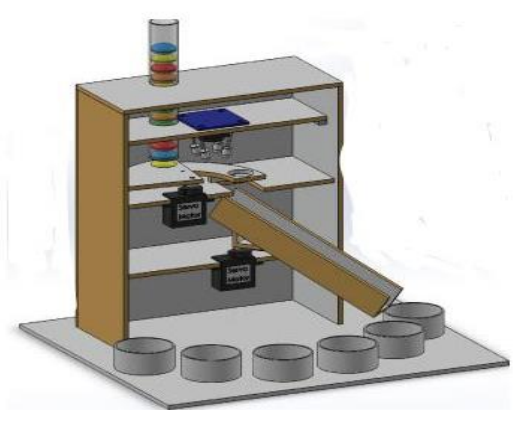

Fig 2: Hardware model of Object Detector

\section{Simulation Results}

A well refined automation system that increases the rate of production will be a perfect and ideal alternative to the conventional manual sorting process. The conventional method of sorting object consumes more manpower. The conventional sorting system ingests more time to sort them. But it leads to certain manual errors such as low accuracy, low efficiency, etc. To reduce fabrication time as well as increasing quality automated systems is being used. The proposed system can sense, sort and count objects based on their colors and to display them in LCD display and stored using Cloud. The proposed object detection model can sort the items of different colors accurately, but when the light conditioning changes, results may vary. The output data is sent to the server and data is visualized using Thing speak platform. These API keys are used in the program as and the data values from the sensors are uploaded to the respective channel whose API key is mentioned. 


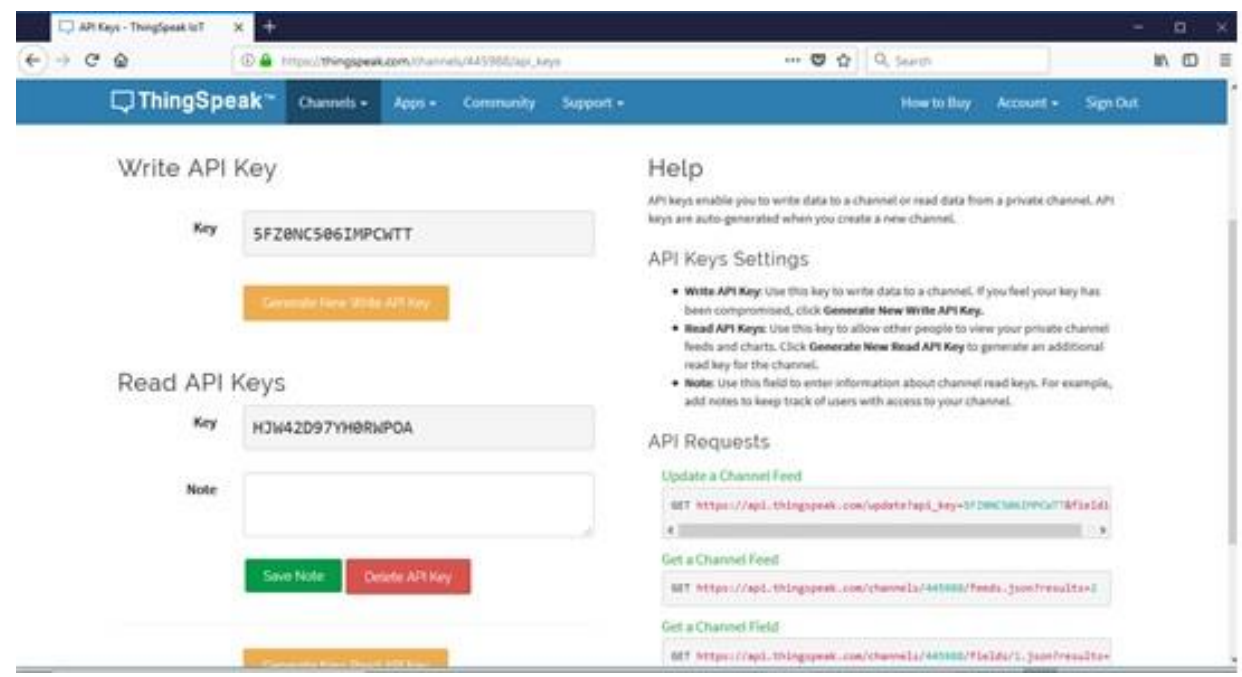

Fig 3: API Channel setting in Thingspeak server

\section{Conclusion}

This color sorting machine says goodbye to handpicking process of sorting the objects of different color reduces the human effort, labor problem in industry and thus efficient economic wise also. With this industrial automation, time can be saved along with quality of work can be improved. Today, we measure the resistance of a resistor in lab with color coding principle. In near future to fasten the process further, multimeters can be employed. But, what if a greater number of resistors are given and asked to sort (Imagine the situation in research lab, where the concerned person has to sort thousands of resistors). This is where color sorting machine can be very useful. In future, by using advanced sensor, based on this principle, we can sort the resistors according to color coding with more advanced programming, which will be very useful in Engineering Colleges. Advanced sensor means which can even detect the color of narrow width.

\section{References}

[1] Felzenszwalb, Pedro F., et al. "Object detection with discriminatively trained part-based models." IEEE transactions on pattern analysis and machine intelligence 32.9 (2009): 1627-1645.

[2] Himanshu Patel, Riya Joy, Selin Macwan, Hardik Modi, "IOT Color Based Object Sorting Machine", International Journal of Applied Engineering Research, vol. 13, no. 10, (2014),0973-4562.

[3] H. K. Patel, P. Verma and S. Ranka, "Design and Development of Coordinate Based Autonomous Robotic Arm", International Conference on Current Trends in Technology, pp. 382-421, 2011.

[4] I. Vojtko, M. Kocisko, M. Janak and V. Fecova, "The New Design of Robot Arm", IEEE 11th International Symposium on Applied Machine Intelligence and Informatics, pp. 53-56, 2013.

[5] Q. Fan, P. Gabbur and S. Pankanti, "Relative Attributes for Large-Scale Abandoned Object Detection," 2013 IEEE International Conference on Computer Vision, Sydney, NSW, 2013, pp. 2736-2743, doi: 10.1109/ICCV.2013.340.

[6] A. Bayona, J. Miguel, and J. Martinez. Comparative evaluation of stationary foreground object detection algorithms based on background subtraction techniques. In AVSS, pages 25-30, 2009.

[7] M. Bhargava, C. Chen, M. Ryoo, and J. Aggarwal. Detection of abandoned objects in crowded environments. In AVSS, 2007.

[8] Q. Fan and S. Pankanti. Modeling of temporarily static objects for robust abandoned object detection in urban surveillance. In AVSS, 2011 